\title{
Von der Raumstadt zur Sozialen Stadt
}

\section{Die Entwicklung problematischer Stadtteile - Beispiel Nordweststadt in Frankfurt am Main}

\section{Therese Neuer-Miebach}

Frequently, districts of German cities that have been built after WW II are problem areas. The social conditions there are more likely to hinder the development of social opportunities rather than promoting them. The quarter called Nordweststadt in Frankfurt on the Main is one example.

Fréquemment, des incidents surviennent dans les quartiers des villes allemandes qui ont été construites après la Seconde Guerre mondiale. Les conditions socio territoriales auraient davantage pour effet de limiter le développement social que de le promouvoir. Le Nord Ouest de Frankfurt-sur-le-Main en est un bon exemple.
Prof. Dr. Therese Neuer-Miebach lehrt an der Fachhochschule Frankfurt am Main Interventionslehre und Konzepte in der Sozialarbeit (vgl. Autorenporträt auf Seite 21).

E-Mail neuer@fb4.fh-frankfurt.de
In vielen deutschen Großstädten stellen gerade die in den Nachkriegsjabren entstandenen Stadtteile problematische Wohnquartiere dar. Die sozialräumlichen Bedingungen in diesem Wohnumfeld scheinen eher dazu angetan, die gesellschaftlichen Chancen von Menschen zu hemmen als sie erhöhen. Die Nordweststadt in Frankfurt am Main ist ein Beispiel.

Die Nordweststadt in Frankfurt am Main ist die gebaute Vorstellung der 1950er und 1960er Jahre von Wohnbedingungen, Geborgensein und Lebensqualität der kleinbürgerlichen städtischen Familie in Erwartung stetiger Verbesserung der sozio-ökonomischen Entwicklungsbedingungen*.

Gründe für den Entwurf der Raumstadt Nordweststadt durch den Frankfurter Architekten Schwagenscheidt waren die kriegsbedingte Zerstörung großer Teile des innerstädtischen Wohnungsbestandes, Bevölkerungszunahme infolge von Flucht und Geburtenanstieg sowie der steigende Wohnflächenbedarf. Bereits 1949 hatte er dieses Konzept entworfen, das nicht nur architektonisch-gestalterische Ideen im Wohnungsbau realisieren wollte. Dahinter stand das gesellschaftspolitische Leitbild einer aufstrebenden liberalen Stadtgesellschaft, die Individualität und Pluralität zulässt - ein $\mathrm{Zu}$ sammenleben unterschiedlicher Schichten im "offenen Raum «, eine Synthese von Verschiedenartigkeit und Vielfalt, von Nähe und Distanz (Kampffmeyer 41, 105).

Dieses Konzept setzt nicht nur ökonomische Aufwärtsentwicklung voraus, sondern auch den aktiven, zielstrebigen Bürger, der seinen Beitrag zum Bruttosozialprodukt erbringt und dafür in den Genuss sozialstaatlicher Sicherung kommt. Die räumliche Nähe unterschiedlicher sozialer Schichten - heute würde man eher von sozialer Mischung sprechen - gehörte zur Vorstellung eines dynamischen
Prozesses des Zusammenlebens im Stadtteil.

In der Frankfurter Nordweststadt als systematisch konstruierte Stadt in der Stadt für mehr als 25.000 Einwohner, wurden zwischen 1962 und 1972 zirka 7.800 Mietwohnungen und rund $800 \mathrm{Ei}-$ genheime gebaut - etwa 100 unterschiedliche Wohnungs- und Hausgrundrisstypen auf einer Fläche von 170 Hektar. Der größte Teil der Wohnungen (mehr als 6.200 Wohneinheiten), die sowohl im Standard als auch in der Größe zukunftsweisende Maßstäbe setzte, entstand im Sozialen Wohnungsbau, öffentlich subventioniert zur Förderung der Wohnungsversorgung breiter Schichten der Bevölkerung.

Zum Konzept des hochwertigen Wohnens in intakter Gemeinschaftlichkeit gehörten die aufgelockerte Mischung unterschiedlicher Gebäudetypen mit eingestreuten großzügigen Grün- und Freiflächen (allein der Martin-LutherKing-Park mit acht Hektar), die Anlage des räumlich konzentrierten Infrastruktur- und Dienstleistungszentrums Nordwestzentrum (zugleich als »bürgerschaftlicher Mittelpunkt « konzipiert; Kampffmeyer 92) und dreier dezentral gelegener sogenannter »Kleine Zentren « sowie ein differenziertes Vorschul- und Schulangebot.

\section{»Frankfurt am Main - Soziale Stadt «}

Es waren insbesondere wohnungswirtschaftliche Interessen an der Verwertung des eigenen Bestandes, die das Bund-Länder-Programm "Stadtteile mit besonderem Entwicklungsbedarf - Die Soziale Stadt « von 1999 angestoßen haben. Auch das darauf basierende kommunale Programm der Stadt Frankfurt am Main »Frankfurt - Soziale Stadt « wurde maßgeblich durch Unternehmen der Wohnungswirtschaft initiiert, die infolge von 
demografischen, sozio-ökonomischen und sozio-kulturellen Veränderungen die Vermietbarkeit ihres eigenen Bestandes gefährdet sahen.

Erklärtes Ziel des Frankfurter Programms ist es, die Wohn- und Lebenssituation zu verbessern, " positive Zukunftsperspektiven von Wohngebieten zu unterstützen « durch »eine Stärkung des sozialen Zusammenhalts sowie die Sanierung des baulichen Zustandes der Häuser und des Wohnumfeldes « (Zuwendungsvereinbarung zwischen dem Magistrat der Stadt Frankfurt und den Trägern des Quartiermanagements, 2005 und Internetauftritt des Programms). Insgesamt gab es seit 1999 zehn derartige Quartiersprojekte in Frankfurt am Main, vier davon sind nach fünfjähriger Laufzeit abgeschlossen. Anfang 2005 wurde die Nordweststadt in das Programm »Frankfurt Soziale Stadt « aufgenommen.

\section{Besonderer Entwicklungs- und Sanierungsbedarf in der Nordweststadt}

Die Programmatik der »Sozialen Stadt Frankfurt « bezieht sich auf sozialstrukturelle und städtebauliche Faktoren. Für die vorliegende Analyse war zu unterscheiden zwischen politischen Annahmen, ökonomischen Erwartungen und empirischen Fakten.

Das heutige Image der Nordweststadt unterscheidet sich wesentlich von dem vor 40 Jahren: anstelle von Aufstiegsorientierung steht heute soziales Risiko für viele der Bewohnerinnen und Bewoh- ner, anstelle von gehobenem Wohnen steht heute eine wenig attraktive Wohnadresse.

Faktum ist, dass das soziale und familienpolitische Leitbild des Konstrukts Nordweststadt und seine städtebauliche Umsetzung den heutigen Realitäten veränderter Lebensbedingungen von Familien und den pluralen Lebensentwürfen und Wohnbedürfnissen nicht mehr entspricht: Heute wohnen in der Nordweststadt weniger, andere und ärmere Menschen, bedingt durch lebenszyklische Entwicklung bei den alteingesessenen und der instabilen sozio-ökonomischen und kulturellen Ausstattung der zugezogenen Haushalte. $\mathrm{Zu}$ den wenigen bekannten Eckdaten sozialstruktureller Veränderung gehören, ähnlich wie in vergleichbaren Gebieten

- die Differenzierung von Erwerbsbiografien (nicht mehr der gut qualifizierte, dauerhaft beschäftigte Haushaltsvorstand ist das Muster für die Sicherung des Familieneinkommens)

- die deutliche Zunahme des Anteils alter deutscher Bewohner (der Anteil der über 65-Jährigen hat seit 1968 insgesamt zugenommen von 6,7 auf 20 Prozent im Jahr 1998 (Schader-Stiftung Datenreport 1998) und

- des Anteils der Menschen mit Migrationshintergrund (der Anteil der Bewohner mit ausländischer Staatsangehörigkeit erhöht sich von drei Prozent im Jahr 1970 auf 25 Prozent im Jahr 1996; während der Anteil der Jugendlichen insgesamt eher rückläufig zu sein scheint, erhöht sich der Anteil Jugendlicher mit Migrationshintergrund er-

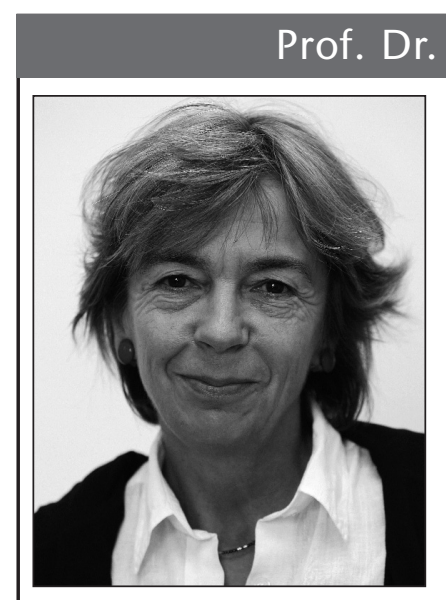

Therese Neuer-Miebach

lehrt seit 1999 am Fachbereich Soziale Arbeit und Gesundheit der Fachhochschule Frankfurt am Main. Sie hat Soziologie, Romanistik und Katholische Theologie studiert. Sie ist Mitglied der Sektion Stadtund Regionalsoziologie der Deutschen Gesellschaft für Soziologie und Mitglied des Nationalen Ethikrates der Bundesregierung. Ihre Forschungsschwerpunkte sind die sozialräumliche Entwicklung, die demokratiepolitische und wohlfahrtsstaatlichen Konzepte der Partizipation, die Begleitforschung zum Projekt "Soziale Stadt « (insbesondere das Teilprojekt »Aktivierung der BewohnerInnen/Verbesserung der BürgerInnenmitwirkung und des Stadtteillebens 2001-2005). Sie verantwortete verschiedene Untersuchungen zur »Sozialen Stadt . Als frühere Mitarbeiterin der Bundesvereinigung Lebenshilfe für Menschen mit geistiger Behinderung beschäftigt sie sich zudem mit den ethischen Aspekten der Biomedizin.

E-Mailneuer@fb4.fh-frankfurt.de

heblich; nach aktuellen Schätzungen des Nachbarschaftsbüros dürften 80 bis 90 Prozent der Kinder einen Migrationshintergrund haben, also mindestens ein Elternteil ausländischer Herkunft sein).

Dieser Befund ist ein Indiz für die bevölkerungsstrukturelle Annahme einer Polarisierung zwischen alten Deutschen und jungen Migranten. Inwieweit dies mittelfristig stadtentwicklungsplanerisch relevant ist, wäre im Rahmen eines integrierten Handlungskonzepts für den Stadtteil zu prüfen. Die empirische Datenbasis hinsichtlich zentraler sozialwissenschaftlicher Risikofaktoren wie Einkommensarmut, niedriges Bildungsniveau, hohe Erwerbslosigkeit und Bevölkerungsfluktuation für die Nordweststadt ist zehn Jahre alt und stellt lediglich Durchschnittwerte im Vergleich $\mathrm{zu}$ anderen statistischen Bezirken der Stadt dar. In der sechsstufigen Risikoskala der Frankfurter Sozialberichterstattung rangiert der Stadtteil auf der zweitniedrigsten Stufe - also kaum ein Indiz für besonderen Entwicklungsbedarf.

Die städtebauliche Struktur der Nordweststadt hat sich kaum verändert. Die befragten Vertreter der Wohnungsbauunternehmen betonen, die Nordweststadt sei keineswegs ein markantes Problemgebiet und die Nachfrage nach Wohnungen dort sei ungebrochen. Zugleich konstatieren sie einen Modernisierungsbedarf im Wohnungsbestand und einen erheblichen Erneuerungsbedarf im Wohnumfeld. Es sind gerade Stimmen aus der Wohnungswirtschaft, die die kommunalpolitische Intervention angeregt haben - die Gefahr des Attraktivitätsverlusts des eigenen Wohnungsbestandes und die öffentlichen Fördermöglichkeiten im Blick.

Von kommunalpolitischem Interesse sollte die Wohnungsbelegung sein. Immerhin läuft bei 80 Prozent aller öffentlich geförderten Wohnungen die Mietpreisbindung aus und damit entfällt die Steuerung der Wohnungsbelegung durch das städtische Wohnungsamt.

Die Aufnahme des mittleren Bereichs der Nordweststadt in das kommunale Programm »Frankfurt - Soziale Stadt" (mit insgesamt über 10.000 Bewohnern, gut zwei Drittel der Gesamtbewohnerschaft) im Jahr 2005 war den Erhebungen zufolge eine politische Entscheidung zur Prävention des Imageverlustes des Stadtteils und zur Garantie der Vermiet- 
barkeit des Wohnungsbestandes. Die sozialen Risiken von Bewohnern sind dabei insofern von Belang als angenommen wird, dass "gute Nachbarschaft « und Aktivitäten im und für den Stadtteil Fühlungsvorteile für Wohnqualität sind.

\section{Quartiermanagement}

Träger des Quartiermanagements im Quartier Nordweststadt ist das Diakonische Werk für die Stadt Frankfurt. Das Nachbarschaftsbüro im Quartier ist mit eineinhalb Stellen - einer Stadtplanerin und einem Pädagogen - ausgestattet.

Quartiermanagement ist ein neues, wenig konturiertes Instrument der kleinräumlichen Koordination und Vermittlung. Im Unterschied zum Bund-LänderProgramm konzentriert das Frankfurter Programm die Funktionen des Quartiermanagements auf die Partizipation der Bewohnerschaft: Verbesserung der Kommunikation ("positive Nachbarschaft " und »selbsttragende Bewohnerorganisationen «), Aktivierung von Kompetenzen und Potenzialen und Anregen von Aktivitäten zur Verbesserung der Wohn-, der Wohnumfeld-Situation und der sozialen Infrastruktur.

Das Ergebnis der Befragung von Bewohner-, Jugend-, Wohnungswirtschafts-, Sozial- und Schulexperten ist ein breites Spektrum an teilweise gegenläufigen $\mathrm{Er}$ wartungen an das Quartiermanagement. Es sind dies zum einen realistische Wünsche: Information über die Möglichkeiten des Programms, Transport von Bewohnerinteressen in die kommunalpolitischen und wohnungswirtschaftlichen Entscheidungsstrukturen, Initiierung von Bewohnerkommunikation und Bewohneraktivität für Quartiersbelange, Einbeziehung von Zielgruppen in die Realisierung von Projekten, Vermittlung von Kontakten zu Wohnungsbaugesellschaften und Kommune und zwischen beiden.

Zum anderen werden Anforderungen artikuliert, die den Zeit-, den Kapazitätsund den Kompetenzrahmen des Quartiermanagements deutlich überschreiten und eher darauf aus zu sein scheinen, eigene Aufgaben zu delegieren oder geeignet sind, das Instrument als solches zu überfrachten. Unter den Prämissen eng begrenzter fachlicher Zuständigkeit, fehlender Einbindung in die politischen Entscheidungsstrukturen und knapp bemes- sener Projektlaufzeit kann es nicht Aufgabe des Quartiermanagements sein, beispielsweise die Entwicklung der Sozialstruktur zu beobachten, das Image des Stadtteils aufzuwerten, längerfristig selbsttragende Bewohnerstrukturen in einem Quartier zu schaffen, in dem die größte Energie der Bewohner in die Sicherung des eigenen Überlebens investiert werden muss, die bescheidenen Projektmittel zielgenau, effektiv und zugleich nachhaltig einzusetzen und außerdem Feuerwehrfunktion zu übernehmen bei der Beseitigung von Parkierungs- und Müllproblemen, beim Umgang mit schwierigen Mietparteien oder bei der Einhaltung der Hausordnung.
Dieser Befund ist jedoch relativ und per se keineswegs repräsentativ für die Gesamtbevölkerung, weil die Auswahl der Befragten erfahrungsgemäß selektiv ist: Es sind aktivere, offenere Personen, die sich bereit erklären, an einer solchen, auf Freiwilligkeit basierenden Untersuchung teilzunehmen. Allerdings bestätigen die Wohnungsunternehmen den Eindruck hoher Wohnzufriedenheit und unterstreichen ihre eigenen Aktivitäten zur »Mieterpflege «. Für sie ist die Nordweststadt kein verlorenes Terrain; vielmehr machen sie erhebliche Entwicklungspotenziale im Stadtteil aus und möchten dazu beitragen, die Bindung der Bewohner an das Quartier zu erhöhen - ein Faktor, der den

\section{"Das Zusammenleben von alten Deutschen und jungen Migranten ist eine der zentralen Herausforderung der Stadtteilplanung von beute"}

Dies kann nur zu Frustrationen oder zum in der Sozialen Arbeit hinlänglich bekannten Burnout führen. Quartiermanagement ist »ein Instrument für strategische Allianzen zur Umsetzung begrenzter, gezielter Handlungskonzepte« (Fachhochschule Frankfurt am Main, Soziale Stadt Nordwest, 98). Daher der eindringliche Appell an die Kommune in der Untersuchung, die zeitlichen, finanziellen und politischen Spielräume des Quartiermanagements realistisch abzustecken. Zugleich die Empfehlung, seine sozialräumliche Reichweite und seine Begrenzungen, etwa hinsichtlich der Ausbildungs- und Beschäftigungschancen von Jugendlichen, der Bildungschancen von Kindern mit Migrationshintergrund oder der Mietpreisentwicklung im Zuge von Modernisierungsmaßnahmen, im Rahmen eines integrierten Handlungskonzepts transparent zu machen.

\section{Wohnen im Quartier: Indizien und Tendenzen}

Viele der befragten Bewohner wohnen sehr lange in der Nordwestdtadt, zwanzig, dreißig Jahre. Die Wohnzufriedenheit ist hoch und die Bereitschaft groß, im und für das Quartier selbst aktiv zu werden.
Marktwert des Wohnstandortes zu verbessern verspricht.

Basis gezielter Empfehlungen zur Aktivierung von Potentialen und Ressourcen für die Quartiersentwicklung ist eine valide Analyse von individuellen Bedürfnissen und gesellschaftlichen Notwendigkeiten.

In unserer Studie hat sich gezeigt, dass eine differenzierte, wenngleich keineswegs widerspruchsfreie Beobachtung der Entwicklungstrends sozialer Risiken am ehesten von den (öffentlichen und freien) sozialen Trägern in der Nordweststadt zu erwarten ist. Sie sind es, die eine steigende Zahl der von öffentlichen Transferleistungen abhängigen Familien, große Wohnraumenge in Großfamilien, insbesondere mit Migrationshintergrund, eine Zunahme von Überschuldung und drohendem Wohnungsverlust, von Suchtproblemen und häuslicher Gewalt bemerken. Bei Kindern und Jugendlichen stellen sie vermehrt Ernährungs- und Gesundheitsprobleme fest. Den Anstieg der Fälle von Erziehungshilfen führen sie hauptsächlich auf Wohnraumenge, mangelnde familiäre Betreuung und schulische Probleme der Kinder und Jugendlichen zurück.

Als grundlegendes Problem wird von allen befragten Gruppen die Integration 
der Bewohner mit Migrationshintergrund genannt - dies obwohl keine akuten Probleme benannt werden und keine aktuellen Statistiken vorliegen. Auch im Rahmen dieser Untersuchung ist es nicht gelungen, Zugang $\mathrm{zu}$ finden $\mathrm{zu}$ den Communities von Migranten. Während Wohnungsunternehmen und soziale Träger auf Toleranz, auf »ausgewogene sozi- ale Mischung « setzen und die kulturelle Vielfalt als Gewinn verstanden wissen wollen, fühlen die Bewohner, die sich zu diesem Thema geäußert haben - es sind dies deutsche Bewohner im Pensionsalter mit langjähriger Wohndauer und enger Quartiersbindung - sich bedroht durch Migranten, weisen diesen pauschal die Verantwortung für Nachbarschaftskon-

\section{Literatur}

ARGEBAU - Leitfaden zur Ausgestaltung der Gemeinschaftsinitiative "Soziale Stadt«. 2. Fassung, 1. März 2000. Deutsches Institut für Urbanistik ( $\mathrm{Hg}$.) (2003): Strategien für die Soziale Stadt. Erfahrungen und Perspektiven. Berlin. dass. (2004): Die Soziale Stadt. Ergebnisse der Zwischenevaluierung. Bewertung des Bund-Länder-Programms. Berlin.

Dilcher, R.; Neuer-Miebach, Th. et. al. (2001): Projekt »Frankfurt am Main Soziale Stadt «. Quartiermanagement in Sossenheim Carl-Sonnenschein-Siedlung. Im Auftrag der AWO-Kreisverband Frankfurt e. V., Frankfurt am Main.

Fachhochschule Frankfurt (Hg.) (2005): Soziale Stadt Nordwest - Bewohneraktivierung. Quartier Nordweststadt. Erarbeitet im Fachbereich Soziale Arbeit und Gesundheit unter Leitung von Neuer-Miebach, Th. . Frankfurt am Main Stadt Frankfurt am Main: Information der Stadt Frankfurt am Main, Amt für Wohnungswesen, über das Projekt »Frankfurt am Main - Soziale Stadt Neue Nachbarschaften ", Internet http://www.Frankfurt-Stadt.de/ses [17.6.2005].

Präsentation der neuen Quartiersgebiete ab 2005 durch Bürgermeister Achim Vandreike, Dezernent für Wohnungswesen, Frankfurt am Main 2005, Internet http://www.stadt-frankfurt.de.

GdW Bundesverband deutscher Wohnungsunternehmen e. V. (1998): Überforderte Nachbarschaften. Zwei sozialwissenschaftliche Studien über Wohnquartiere in den alten und neuen Bundesländern. Berlin.

Häußermann, H.; Kronauer, M.; Siebel, W. (Hg.) (2004): An den Rändern der Städte. Frankfurt am Main.

Hessisches Ministerium für Wirtschaft, Verkehr und Landesentwicklung (Hg.)
(2000): Hessische Gemeinschaftsinitiative Soziale Stadt (HEGISS). Wiesbaden.

Kampffmeyer, H. (1968): Die Nordweststadt in Frankfurt am Main. Frankfurt am Main.

Neuer-Miebach, Th.; Blanc, M. (2005): Überforderte Programme für überforderte Nachbarschaften: Reflexionen über den Partizipationsgehalt der Politique de la Ville und des BundLänder-Programms "Soziale Stadt". Arbeitskreis Stadterneuerung in $\mathrm{Zu}$ sammenarbeit mit dem Institut für Stadt- und Regionalplanung der Technischen Universität Berlin (Hg.): Jahrbuch Stadterneuerung 2004/05. Berlin, 311-327.

Neuer-Miebach, Th.; Schneider, J.; Pfeiffer, A. (2004): Evaluation: Aktivierung der BewohnerInnen/Verbesserung der BürgerInnen-Mitwirkung und des Stadtteillebens. Begleitforschung Soziale Stadt Hessen HEGISS, Frankfurt am Main.

Riege, M.; Schubert, H. (Hg.) (2002): Sozialraumanalyse. Grundlagen - Methoden - Praxis. Opladen.

Schader-Stiftung (Hg.) (1998): Datenreport Nordweststadt. Einwohner und Wohnungsbestand. Demonstrativprojekt Demographischer Wandel. Darmstadt. dies. (Hg.) (1999): 2. Datenreport Nordweststadt. Bewohner von Eigenheimen. Demonstrativprojekt Demografischer Wandel. Darmstadt.

Thole, W.; Closs, P. et al. (Hg.) (2005): Soziale Arbeit im öffentlichen Raum. Wiesbaden.

http://www.frankfurt-sozialestadt.de http://www.sozialestadt.de http://www.sozialestadt.de/veroeffentlichungen/newsletter/

http://www.sozial-raum-management.de http://www.stadtteilarbeit.de

Kontakt zum Nachbarschaftsbüro Nordweststadt:E-Mailnordweststadt@frankfurtsozialestadt.de flikte und Hausfrieden zu und stellen die unmissverständliche Forderung nach Wohlverhalten (dies wird gemessen an der Einhaltung der Hausordnung) und Anpassung der »Fremden « an die »deutschen Verhältnisse « (»Zwang zur Anpassung «).

Daher scheint die Empfehlung »Integration von Migranten « (8.I.4) zunächst utopisch zu sein. Immerhin ist es dem Quartiermanagement inzwischen gelungen, das Angebot an Deutschkursen für Eltern von Migrantenkindern auszuweiten, um die Integrationschancen von Eltern, Kindern und Jugendlichen zu verbessern. Die jüngste Gründung eines »Internationalen Vereins für Kinder und ihre Eltern « zielt als ein weiterer Schritt auf eine interkulturelle Verständigung über Elternkompetenz, über familiäre Funktionen und öffentliche Entlastungsund Unterstützungsangebote sowie auf die Erleichterung des Zugangs zu Bildung, Ausbildung und auf gesellschaftliche Teilhabe.

Hauptanliegen aller Befragten ist die Verbesserung der Situation der im Quartier wohnenden Jugendlichen. Der Akzent wird je nach Blickwinkel auf Wohnen, Freizeit, Kriminalität bzw. auf Schule, Ausbildung und Beschäftigung gesetzt.

Die Wohnbedingungen des Großteils der Kinder und Jugendlichen im Quartier werden als hinderlich für die schulischen Leistungen und für die Entwicklung von Selbständigkeit eingestuft. Daraus resultieren Empfehlungen zum Abbau der Überbelegung von Wohnungen und zur gezielten Bereitstellung von Wohnungen für Jugendliche und junge Erwachsene mit sozialpädagogischem Betreuungsangebot in der Übergangsphase.

Dabei kann auf positive Erfahrungen in der Nordweststadt zurückgegriffen werden. Dieser Optimismus wurde inzwischen konterkariert durch zwei Änderungen des SGB II bei der Grundsicherung von Arbeitssuchenden: die generelle $\mathrm{Zu}$ rechnung von Personen bis zur Vollendung des 25.Lebensjahres zur Bedarfsgemeinschaft der Eltern und die deutliche finanzielle Einschränkung dieses Personenkreises beim Umzug in eine eigene Wohnung. Die negativen Auswirkungen dieser Rücknahme wohl bedachter sozialpolitischer Regelungen werden erst längerfristig spürbar und dann, so ist zu befürchten, eher individuell zugerechnet werden. 
Auch wenn im statistischen Durchschnitt der Besatz mit Jugendzentren und Sportflächen als gut zu bezeichnen ist, betonen die Befragten die Notwendigkeit, differenzierter auf die Bedürfnisse von Teilgruppen, wie beispielsweise Mädchen mit Migrationshintergrund, einzugehen und diese selbst in die Umsetzung von gezielten Angeboten einzubeziehen. Die Professionellen der Sozialen Arbeit zeigen sich bereit, auch selbst mehr in die Stadtteiljugendarbeit $\mathrm{zu}$ investieren, Projekte ins Leben $\mathrm{zu}$ rufen und Initiativen $\mathrm{zu}$ unterstützen. Allerdings wird ausdrükklich darauf hingewiesen, dass dies lediglich flankierende Maßnahmen und keine wirkliche Lösung gesamtgesellschaftlicher Probleme sein könnten.

Die Reichweite des Bund-Länder-Programms und der kommunalen Programme »Soziale Stadt « hinsichtlich der zentralen Handlungsfelder Bildung, Beschäftigung und Migration ist strukturbedingt begrenzt. Auf Quartierebene, im sogenannten Sozialraum, sind die gesamtgesellschaftlichen sozio-ökonomischen Rahmenbedingungen nicht veränderbar. Hinzu kommt eine nachhaltige Veränderungsresistenz in der ressortfi- xierten kommunalen Administration, die, wie bundesweite und vergleichende Untersuchungen im Rahmen der Sozialen Stadt zeigen (DifU 2004), nur in seltenen Fällen durch »integrierte Handlungskonzepte « aufzubrechen sind.

Dies gilt im übrigen auch für die Durchsetzbarkeit von Bewohnerpartizipation gegenüber kommunalpolitischen Entscheidungsstrukturen (Neuer-Miebach/Blanc 2004). Dennoch stellt das Programm - gerade auch im Bewusstsein und in Kenntnis seiner tatsächlichen Spielräume - eine Chance dar für engagierte Kommunen. Daher empfiehlt die Untersuchung beispielsweise kurzfristig die Nutzung der kommunalen Wohnungsbelegungsrechte. Darüber hinaus sollte die Bewohnerstrukturentwicklung in diesem Quartier (wie in anderen Quartieren der Sozialen Stadt) genau beobachtet und analysiert werden, um aktionsfähig zu sein zum Zeitpunkt, zu dem die Mietpreisbindungen auslaufen.

Diese Empfehlung wurde inzwischen partiell aufgegriffen in Überlegungen zu neuen freien Vereinbarungen zwischen der Stadt Frankfurt am Main und Wohnungsunternehmen über die gezielte »gemischte « Belegung einzelner Wohngebäude. Zudem ist es Aufgabe der Kommune, die Wohnungsversorgung von einkommensschwachen Haushalten sicherzustellen, für die die erwartbar deutlich höheren Mietpreise nach Auslaufen der Mietpreisbindung nicht bezahlbar sind und die sich auf dem freien Wohnungsmarkt kaum behaupten können.

Ein Resultat der Untersuchungsergebnisse zum Quartiermanagement ist die Empfehlung, das zuständige Sozialrathaus als dauerhaften sozialen Dienstleister frühzeitig in die Begleitung und Organisation der sozialen Initiativen, die auf Nachhaltigkeit angelegt sind, einzubeziehen - soweit dies von den Bewohnern gewünscht wird.

Aus sozialwissenschaftlicher Sicht wird der Kommune nahe gelegt, die einzelnen Projekte und Maßnahmen des Quartiermanagements in ein noch zu entwickelndes integriertes Handlungskonzept für die Nordweststadt einzubinden. Dazu gehört auch ein abgestimmtes Verfahren über die Verteilung der Mittel für das Quartiermanagement und der investiven Mittel für Wohnungsbau, Wohnungsmo-

\section{Die Nordweststadt}

ist eine Großsiedlung in Frankfurt am Main, die 1962 bis 1972 auf der grünen Wiese entstand. (In sozialarbeiterischen Fachkreisen ist die Siedlung bundesweit als früherer Standort des Deutschen Vereins für private und öffentliche Fürsorge und des Instituts für Sozialarbeit und Sozialpädagogik ISS bekannt.)

Rund 7.800 Mietwohungen, davon 80 Prozent öffentlich gefördert, und 800 Eigenheime wurden auf einer Fläche von 170

Hektar errichtet. Alle Gebäude sind exakt in Nord-Süd- oder Ost-West-Richtung ausgerichtet. Die Fußwege verlaufen getrennt vom Fahrverkehr. Die Siedlung ist durch große Grünflächen in Nachbarschaften gegliedert. Im Jahre 1968 wurde die Nordweststadt an die erste Linie der neuen U-Bahn in Frankfurt am Main angeschlossen. So konnten die Einwohner in kurzer Zeit die Innenstadt erreichen.

Die Schaffung eines differenzierten Vorschul- und Schulangebotes (so beispielsweise die Ernst-Reuter-Schule, eine der ersten integrierten Gesamtschulen in der Bundesrepublik Deutschland) sowie konzentrierte Infrastruktur- und Dienstleistungen im Nordwestzentrum und in drei »Kleinen Zentren « gehörten zum Konzept, das eine reine Schlafstadtatmosphäre vermeiden und zugleich Kommunikations- und Identifikationsräume bereit stellen wollte.

Der mittlere Bereich der Nordweststadt, in dem das Nordwestzentrum liegt, wurde als Quartier der Sozialen Stadt ausgewählt (inzwischen erweitert um das »Kleine Zentrum Thomas-Mann-Straße «). Dort wohnen über 40 Prozent der Bevölkerung der Nordweststadt.
Die Bewohnerschaft hat seit 1970 um mehr als 5.000 Einwohner abgenommen; sie ist bunter geworden: Lag der Anteil der nichtdeutschen Bewohner 1970 bei drei Prozent, so sind es heute 27 Prozent. Und sie ist älter geworden: Waren 1968 nur 6, 7 Prozent der Bewohner 65 Jahre alt und älter, so sind es im Jahr 2005 bereits Prozent.

Aus den Wünschen, die in den Befragungen genannt wurden, hat die Untersuchung zentrale Empfehlungen abgeleitet: die Verbesserung des Wohnumfeldes (Spielflächen und Parken), die Bereitstellung von Wohnraum für junge Erwachsene, die Entwicklung und Umsetzung eines Konzepts für altengerechtes Wohnen und begleitetes Umzugsmanagement, sozialpolitische Vorsorge für den Wegfall der Mietpreisbindung in absehbarer Zeit, die Schaffung eines Treffpunktes für alteingesessene Bewohner, ein kooperatives Stadtteilschulkonzept, die stadtteilspezifische Prüfung des Freizeitangebotes für Kinder und Jugendliche.

Mittlerweile hat das Quartiersmanagement einige Projekte in Gang gesetzt, beispielsweise einen Teenie-Club für Kinder zwischen 12 und 14 Jahren, für Jugendliche ein Theaterprojekt und ein Kursangebot zum Erwerb der Sportübungsleiterlizenz, einen Einkaufsservice für ältere Menschen; Wohnumfeldverbesserungen in Zusammenarbeit mit den Wohnungsunternehmen wurden begonnen und ein Arbeitskreis gegründet, der sich um Bildung im Stadtteil - das Konzept für Stadtteilschule kümmern wird. 
dernisierung und Wohnumfeldgestaltung. Empfohlen wird auch die Inanspruchnahme von Bundesmitteln und Mitteln aus einschlägigen Fonds der Europäischen Union.

Die Entwicklung in den zeitgleich laufenden Projekten der Sozialen Stadt in Frankfurt am Main sollte koordiniert und vergleichend beobachtet werden. Nachhaltige Wirkungen des kommunalen Programms werden sich nur erzielen lassen, wenn politischer Konsens hergestellt werden kann über die Rolle und Funktion dieses Stadtteils im städtischen Gesamtgefüge und seines Stellenwerts innerhalb der Stadtentwicklungsplanung.

\section{Perspektiven}

Ziel der ursprünglichen stadtplanerischen Konzeption für die Nordweststadt in Frankfurt am Main war es, einen lebendigen, entwicklungsfähigen Stadtteil zu schaffen mit unterschiedlichen Menschen, die »das gesellschaftliche $\mathrm{Zu}$ sammenleben anregen und befruchten « (Kampffmeyer 109).

Dieses unter anderen sozio-ökonomischen Vorzeichen entworfene Leitbild sollte nichts von seiner sozialpolitischen Prägnanz verloren haben. Die zentrale Herausforderung der Stadt(teil)entwicklungsplanung heute ist die transkulturelle Stadtgesellschaft mit alternden Deutschen und jungen Migranten. Insbesondere Jugendliche sind es, die von den zentralen Problemen und Handlungsfeldern der Sozialen Stadt elementar betroffen sind: Bildung, Beschäftigung, Migration.

Die sozialräumlichen Bedingungen in Quartieren wie die Nordweststadt scheinen eher dazu angetan, die gesellschaftlichen Chancen junger Menschen zu hemmen als zu erhöhen. Diese benachteiligenden Faktoren, nicht die Jugendlichen selbst, sind das Störpotenzial, das es zu entschärfen gilt.

\section{Fazit}

Städtebau und Sozialstrukturentwikklung stellen auch heute noch keinen Automatismus mit aufsteigender Tendenz dar.

"Bonding or bridging « des Sozialraums? Gerade angesichts globaler Veränderungen der nationalstaatlichen Ek- kpfeiler gesellschaftlicher Entwicklung sind eine enge Fixierung des Programms Soziale Stadt (auf Bundes- wie auf kommunaler Ebene) und eine stadtsoziologische Konzentration auf den Sozialraum Quartier diskussionsbedürftig.

* Der Artikel basiert auf den Ergebnissen einer empirischen Untersuchung des Handlungsbedarfs für das Quartiermanagement in der Nordweststadt Frankfurt am Main, durchgeführt von der Fachhochschule Frankfurt am Main im Jahre 2005 im Auftrag des Diakonischen Werks. Themen, Potenziale und Ressourcen für die Mitwirkung und (Selbst-) Aktivität der Bewohnerschaft sollten aufgezeigt und Empfehlungen gegeben werden für Strategien, Maßnahmen und Projekte. Fokus der Studie waren Wohnen und Leben im Quartier, insbesondere für Jugendliche. Es wurden primär- und sekundäranalytische, qualitative und quantitative Instrumente empirischer Sozialforschung eingesetzt (Fachhochschule Frankfurt am Main, Soziale Stadt Nordwest - Bewohneraktivierung, Frankfurt am Main 2005).

\section{Das neue Standardwerk}

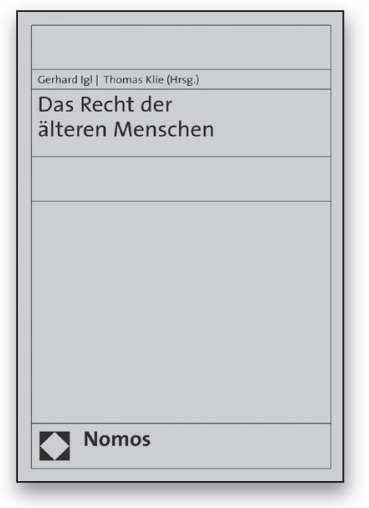

\section{Das Recht der älteren Menschen}

Herausgegeben von Prof. Dr. Gerhard Igl, Universität Kiel und Prof. Dr. Thomas Klie, Ev. Fachhochschule Freiburg

2007, ca. 265 S., brosch., ca. 34,-€, ISBN 978-3-8329-2239-9

Erscheint Februar 2007

Die Zahl älterer Menschen wächst in Deutschland und überall in Europa. Das vorliegende Buch macht deutlich, wie sich der demografische Wandel auf das Recht auswirkt. Namhafte juristische Autoren zeigen die vielfältigen Fragestellungen auf, die ältere Menschen betreffen und die Rechtswissenschaft herausfordern. Das Werk richtet sich vor allem an Rechtsanwälte, Beratungsinstitutionen im Sozial- und Gesundheitswesen, Verbraucherschutzverbände sowie Einrichtungen und Dienste für ältere Menschen.

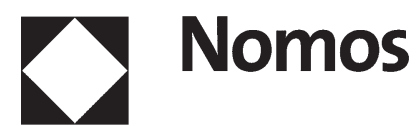

